\title{
Aportes al modelo hidrogeológico conceptual de la Cuenca del Río Quequén Grande en su límite suroccidental
}

\author{
Maria Ximena Solana ${ }^{(1,2)}$; Orlando Mauricio Quiroz Londoño ${ }^{(1,2)}$; Pablo Weinzettel ${ }^{(3)}$; \\ Flavia Donna ${ }^{(1,2)}$
}

(1) Instituto de Geología de Costas y del Cuaternario. Facultad de Ciencias Exactas y Naturales. CIC Universidad Nacional de Mar del Plata. Funes 3350, 7600 Mar del Plata, Argentina. ximesolana@hotmail.com; flaviadonnar@gmail.com

(2) IIMyC Instituto de Investigaciones Marinas y Costeras. Rodríguez Peña 4046, 7600 Mar del Plata, Argentina. qlondon@mdp.edu.ar

(3) IHLLA Instituto de Hidrología de Llanuras (UNCPBA, CIC, Mun. Azul). República de Italia 780, 7300 Azul, Argentina. paw@ihlla.org.ar

\section{RESUMEN}

La Cuenca del Río Quequén Grande es una de las más extensas y productivas del sudeste bonaerense. Sus nacientes se encuentran ubicadas en las serranías deTandilia, que constituyen el área de recarga preferencial del acuífero Pampeano en la región. Este acuífero, somero y de tipo libre, presenta injerencia sobre cursos de agua de carácter efluente. En su límite suroccidental, su morfología se presenta fuertemente asimétrica y destaca la presencia de numerosos humedales. Este fenómeno es asociado a rocas paleozoicas que afloran saltuariamente y constituyen lo que se ha considerado como el basamento hidrogeológico del acuífero Pampeano. Desde el punto de vista geoquímico, en esta zona se diferencian dos tipos de laguna relacionados con su contenido en cloruros, pudiendo relacionarse el grupo de alto contenido en este anión con flujos regionales ascendentes. El objetivo del presente trabajo consiste en aportar nueva información al modelo hidrogeológico conceptual que se tiene para la zona mediante el análisis de captaciones de agua superficial y subterránea. El mapa piezométrico reveló dos direcciones preferenciales en el flujo subterráneo. En el análisis fisicoquímico se observó que la mayoría de las muestras de agua subterránea presenta una composición bicarbonatada sódico-magnésica. Por otro lado, las muestras de agua obtenidas de lagunas presentaron una composición bicarbonatada sódica, distinguiéndose un grupo principal con mayor contenido en cloruros. La correlación entre las desviaciones isotópicas de ambos tipos de muestras sugiere la presencia de recarga de algunas lagunas procedente de las zonas de recarga regional en el sistema serrano de Tandilia.

Palabras Clave: Acuífero Pampeano, hidrogeología, relación humedales/agua subterránea, hidrogeoquímica, isótopos.

\section{Contributions to the conceptual hydrogeological model of the Quequén Grande River Basin at its southwestern limit}

\begin{abstract}
The Quequén Grande River Basin is one of the most extensive and productive areas of the south-east of Buenos Aires Province. Its birth is located in the mountain range ofTandilia, which constitutes the preferential recharge area of the Pampean aquifer in the region. This shallow, free type aquifer interferes with effluent watercourses. At its southwestern limit, its morphology is strongly asymmetrical and highlights the presence of several wetlands. This phenomenon is associated with the presence of Paleozoic rocks that outcrop occasionally and constitute the hydrogeological basement of the Pampean aquifer. Geochemically, two different groups of wetlands are distinguished in this area related to their chloride content, and the one with a high content in this anion may be related to groundwater upward flows. The aim of this study is to provide new information to the conceptual hydrogeological model for the area through the analysis of surface and ground-
\end{abstract}


water catchment areas. Two preferential directions in the groundwater flow were identified. In the physicochemical analysis of groundwater samples, a bicarbonate sodium-magnesium composition was observed. On the other hand, wetland water samples had a bicarbonate sodium composition, distinguishing a main group with higher chloride content. The correlation between the isotopic deviations of both types of samples suggests the occurrence of recharge of some wetlands from the mountain range of Tandilia.

Keywords: Pampean aquifer, hydrogeology, wetlands/groundwater relationship, hydrogeochemistry, isotopes.

\section{Introducción}

La Cuenca del Río Quequén Grande (CROG) es una de las cuencas hidrográficas más importantes de Argentina. Con una extensión de casi $10000 \mathrm{~km}^{2}$, en ella se desarrollan principalmente actividades económicas de origen agropecuario, ubicándose en su desembocadura al Océano Atlántico uno de los mayores puertos exportadores nacionales. Geográficamente, la CROG se encuentra localizada en la cuenca sedimentaria de Claromecó, ubicada entre los sistemas serranos de Ventania y Tandilia, siendo el sector sur de esta última el área de recarga preferencial de la cuenca. Estos elementos estructurales positivos están formados por rocas ígneo-metamórficas de origen Precámbrico y sedimentitas paleozoicas, mientras que en la llanura se desarrolla una cubierta cenozoica de sedimentos principalmente limosos y limo-arcillosos intercalados por delgadas capas de arena que se acumularon durante el Pleistoceno-Holoceno por la acción eólica y fluvial (figura 1).

Desde el punto de vista hidrogeológico, la cubierta cenozoica conforma el acuífero Pampeano (Sala, 1975), que se apoya sobre las sedimentitas paleozoicas que constituyen el basamento hidrogeológico del área (Llambías y Prozzi, 1975), siendo la principal fuente de abastecimiento de agua para la región. EI acuífero, somero y de tipo libre, actúa al mismo tiempo como fuente de recarga de humedales y presenta gran injerencia sobre arroyos y ríos, en su mayoría con comportamiento efluente a lo largo de su recorrido (Kruse et al., 1997; Martínez et al., 2007; Quiroz Londoño et al., 2008; Quiroz Londoño et al., 2009). Mientras que en el río Quequén Grande el flujo superficial se produce a una velocidad de $15,29 \mathrm{~m}^{3} / \mathrm{s}$, el flujo promedio en sus principales tributarios es de 6,079 $\mathrm{m}^{3} / \mathrm{s}$ en el arroyo Pescado Castigado, 2,27 $\mathrm{m}^{3} / \mathrm{s}$ en el Quequén Chico y $0,75 \mathrm{~m}^{3} / \mathrm{s}$ en el arroyo Quelancitá, entre otros (Quiroz et al., 2015). En lo que se refiere al flujo de agua subterráneo, éste se muestra generalmente alineado con el drenaje superficial (Quiroz et al., 2015).

En las últimas décadas se han llevado a cabo numerosos estudios con isótopos ambientales con el propósito de estudiar el ciclo hidrológico del agua (Kendall y McDonnell, 2011). Particularmente, su utilización ha contribuido a la comprensión de los procesos hidrológicos que tienen lugar en el agua subterránea, tales como la dirección del flujo subterráneo, la recarga o interacciones con el agua superficial, entre otros (Gat,
1996; Gibson et al., 2005). En este contexto, Quiroz et al. (2015) realizaron la caracterización isotópica de la CROG, reportando valores de $\delta^{2} \mathrm{H}$ y $\delta^{18} \mathrm{O}$ para el agua subterránea próximos a un valor promedio de $\delta^{18} \mathrm{O}=$ $-5.27 \%$ y $\delta^{2} \mathrm{H}=-30.9 \%$, mientras que para el agua de lluvia se reportan rangos comprendidos entre -15.13 y $3.21 \%$ on $\delta^{18} \mathrm{O}$ y entre -114.1 y $18.7 \%$ en $\delta^{2} \mathrm{H}$, obteniendo a partir de estos últimos la línea meteórica local (LML) de la región.

Morfológicamente, la CRQG se presenta fuertemente asimétrica en su límite suroccidental, que constituye una divisoria de aguas caracterizada por la presencia de numerosos humedales, llevando a Martínez (2007) a denominarla como "zona de divisorias con lagunas". En muchos de estos espejos de agua se registran valores de conductividad eléctrica (CE) elevados (Solana et al., 2016). Al mismo tiempo, en el agua subterránea se produce un aumento de la CE en el sentido del río Quequén Grande (Quiroz et al., 2015). Desde el punto de vista topográfico, los humedales se encuentran ubicados en una región más elevada, siendo este levantamiento el que le confiere a la cuenca su asimetría, llevando al río Quequén Grande a recibir todos sus afluentes desde la margen izquierda. Este fenómeno ha sido explicado por Cortizo e Isla (2000) como el resultado de un proceso de captura fluvial de origen geomorfológico, que implica que con anterioridad al mismo existía una red de flujo subparalela. Según estos autores, cursos como los del Quequén Chico continuaban con sentido norte-sur y desembocaban directamente en el océano Atlántico antes de la captura fluvial, atribuida por Martínez et al. (2011) a un control neotectónico del relieve. Asociado a esto, en el límite suroccidental de la CRQG Llambías y Prozzi (1975) reportan la aparición de rocas de basamento en superficie que atribuyen al sistema de Ventania, lo que implica la existencia de un elemento estructural positivo entre estos sistemas serranos. Según Sala (1975), estos afloramientos se corresponderían a rocas del basamento hidrogeológico impermeable que podrían estar actuando como barrera hidráulica para el flujo de agua subterráneo.

Con el propósito de esclarecer el comportamiento hidrológico del límite suroccidental de la CROG, se seleccionaron $3000 \mathrm{~km}^{2}$ de un área dominada por la presencia de humedales con diferentes características fisicoquímicas e isotópicas, abarcando una porción de la CRQG y de las cuencas contiguas. Geográficamente, la zona de estudio se encuentra localizada entre los paralelos $37^{\circ} 52^{\prime} 49^{\prime \prime}$ y $38^{\circ} 21^{\prime} 48^{\prime \prime}$ de latitud sur y entre 


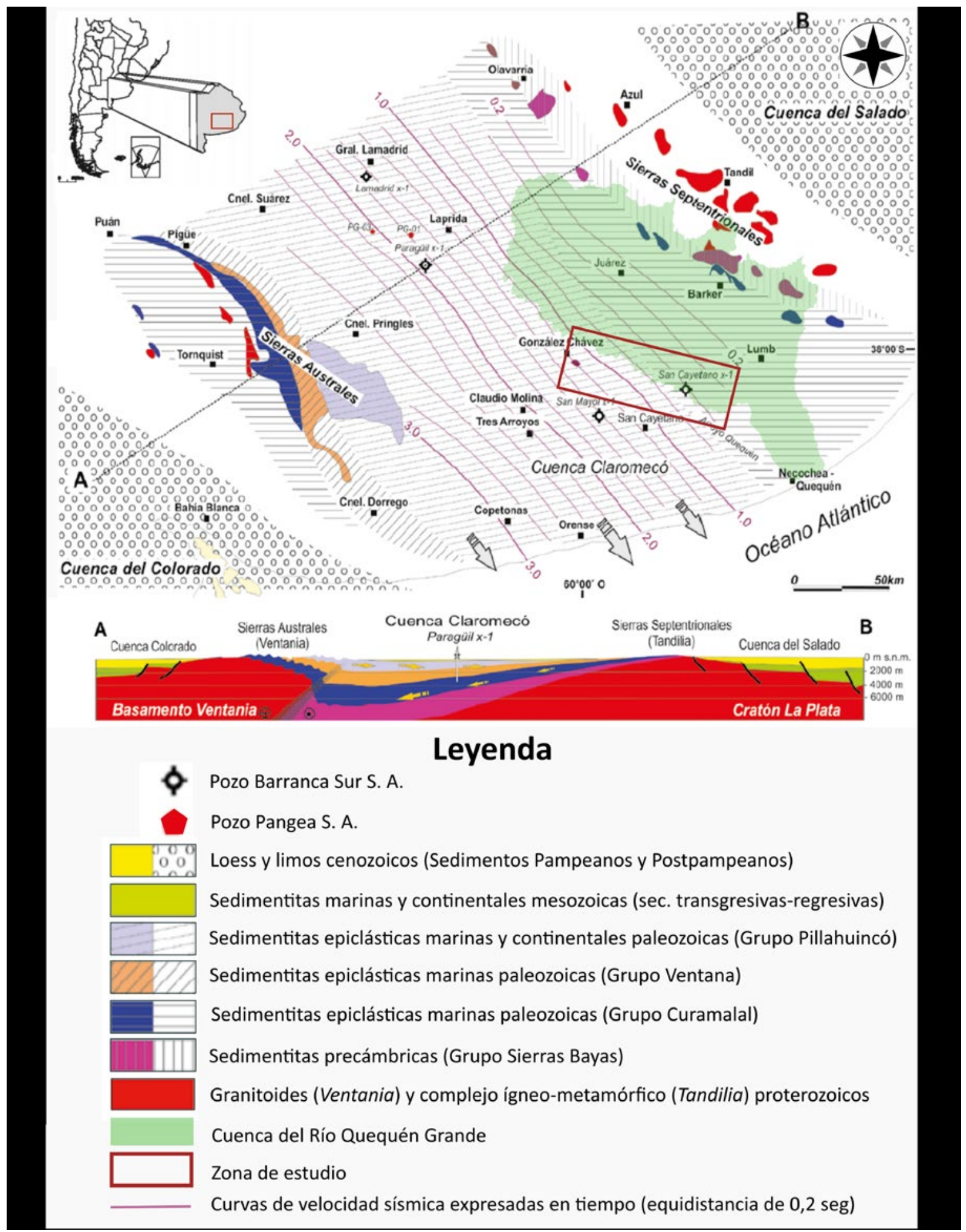

Figura 1. Esquema geológico-estructural de la zona de estudio expresado en curvas de velocidad sísmica, con plano de referencia a $150 \mathrm{~m}$ (arriba) y transecta idealizada de la cuenca de Claromecó (abajo). Se ha destacado en verde la CROG (tomado de Roselló 2016, modificado de Lesta y Sylwan 2005).

Figure 1. Geological-structural scheme of the study area expressed in seismic velocity curves with reference plane at $150 \mathrm{~m}$ (above) and idealized transect of the Claromecó basin (below). The Quequén Grande River Basin (QGRB) has been highlighted in green (taken from Rosello 2016, modified from Lesta and Sylwan 2005). 


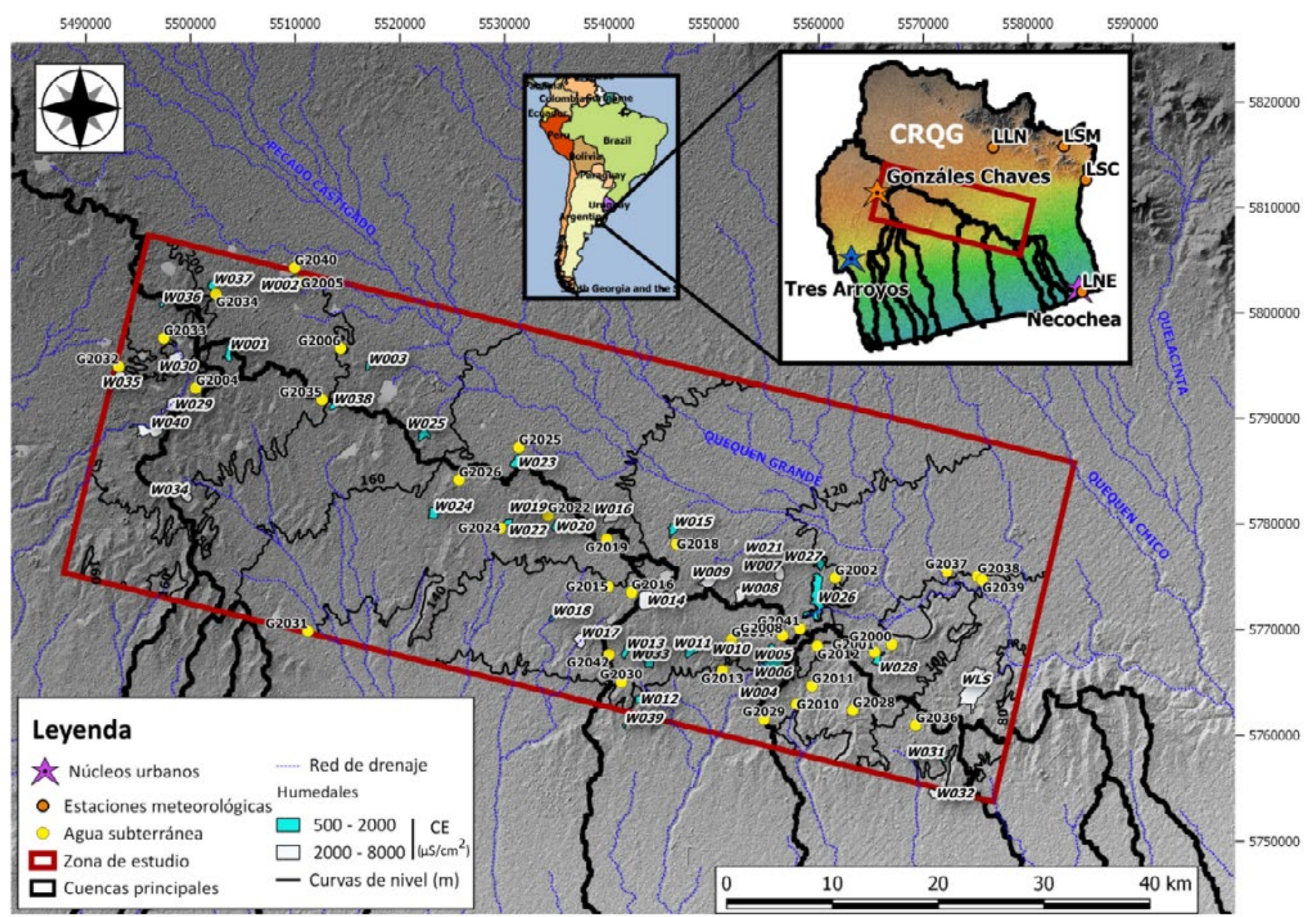

Figura 2. Localización del área de estudio. Arriba: Ubicación de las estaciones meteorológicas y principales núcleos urbanos. Se ha señalado la CROG. Abajo: Puntos de muestreo de agua superficial y subterránea.

Figure 2. Geographic position of the studied area. Above: Location of meteorological stations and main urban centers. The QGRB has been highlighted. Below: Surface and groundwater sampling points.

los meridianos $59^{\circ} 2^{\prime} 36^{\prime \prime}$ y $60^{\circ} 8^{\prime} 17^{\prime \prime}$ de longitud oeste (figura 2). Mediante el uso de técnicas hidrogeológicas, geoquímicas e isotópicas se pretende determinar la relación existente entre los cuerpos de agua superficial localizados en este positivo estructural y el flujo subterráneo, integrándolos al modelo hidrogeológico conceptual que se tiene para la CRQG.

\section{Materiales y métodos}

Se realizaron tres campañas de toma de muestras entre los meses de septiembre de 2015 y noviembre de 2016, en las que se inventariaron un total de 41 puntos de agua superficial y 35 puntos de agua subterránea, registrándose 22 datos de nivel freático. Las muestras obtenidas en cada uno de los puntos inventariados fueron analizadas en el laboratorio de hidrogeoquímica e hidrología isotópica del Instituto de Geología de Costas y del Cuaternario (IGCyC). Las medidas de conductividad eléctrica (CE), $\mathrm{pH}$ y temperatura fueron tomadas in situ y la georreferencia- ción de cada uno de los puntos analizados fue realizada mediante un GPS Garmin eTrex Vista. Toda la información generada fue almacenada en el Sistema de Información Geográfica (SIG) del Grupo de Hidrogeología del IGCyC de la Universidad Nacional de Mar del Plata (UNMdP).

Para la elaboración del mapa piezométrico se consultaron varias fuentes de datos pertenecientes a las siguientes publicaciones:

- Base de datos de Aguas subterráneas perteneciente al Grupo de Hidrogeología de la UNMdP.

- Datos publicados en el Proyecto BID 1201 OC/ AR PICT 08-4675 denominado "Sustentabilidad del riego suplementario en el sudeste de la provincia de Buenos Aires".

- Datos publicados durante el Programa integral de gestión sustentable de los recursos hídricos del partido deTres Arroyos, recopilados en el documento 9 denominado "Hidrogeología del partido de Tres Arroyos, provincia de Buenos Aires"

En el SIG del Grupo de Hidrogeología se compila 
la información hidroclimatológica de un gran número de estaciones ubicadas en el sureste bonaerense, así como parámetros fisicoquímicos e isotópicos del agua tanto superficial como subterránea, con registros desde 1973 a la actualidad. Algunos de los pozos en los que se toman datos de nivel freático son monitoreados en continuo, por lo que se calculó el valor promedio para utilizarlo en la elaboración del mapa piezométrico de la zona. Se utilizaron 46 datos de nivel pertenecientes a esta base. Asimismo, los 22 datos de nivel piezométrico obtenidos en las campañas de campo realizadas entre septiembre de 2015 y noviembre de 2016 fueron incorporados a la misma. Por otro lado, en la publicación perteneciente al partido de Tres Arroyos se recopila un total de 178 datos de nivel piezométrico, todos ellos obtenidos en pozos censados entre el 23 de enero y el 10 de febrero del año 2006 en la cuenca homónima. En lo que se refiere a los datos publicados en el Proyecto BID 1201 OC/AR PICT 08-4675, éstos fueron obtenidos durante una campaña realizada en el año 2001, con densidad de muestreo de 1 punto cada $16 \mathrm{Km}^{2}$, utilizados en la elaboración de la hoja hidrogeológica $\mathrm{N}^{\circ} 14$ perteneciente al río Quequén. La información recopilada se integró para la elaboración del mapa piezométrico de la zona de estudio.

Para la caracterización del agua de lluvia se analizaron los datos de cuatro de las estaciones pluviométricas ubicadas en la CROG, tanto en la zona de recarga como en su desembocadura (figura 2). Estas estaciones forman parte de una red de monitoreo del grupo de hidrogeología de la UNMdP, y en ellas se toman muestras periódicamente desde el 2004.

En el laboratorio se determinó la dureza total y la composición en cationes $\left(\mathrm{Ca}^{2+}, \mathrm{Mg}^{2+}, \mathrm{Na}^{+}\right.$y $\left.\mathrm{K}^{+}\right)$y aniones $\left(\mathrm{Cl}^{-}, \mathrm{CO}_{3}^{2-}, \mathrm{HCO}_{3}^{-}\right.$y $\left.\mathrm{SO}_{4}{ }^{2-}\right)$ mayoritarios de las muestras de agua superficial y subterránea. Para las determinaciones químicas se siguió la metodología detallada en APHA (1998). La información hidroquímica obtenida fue analizada con el programa AQUA-

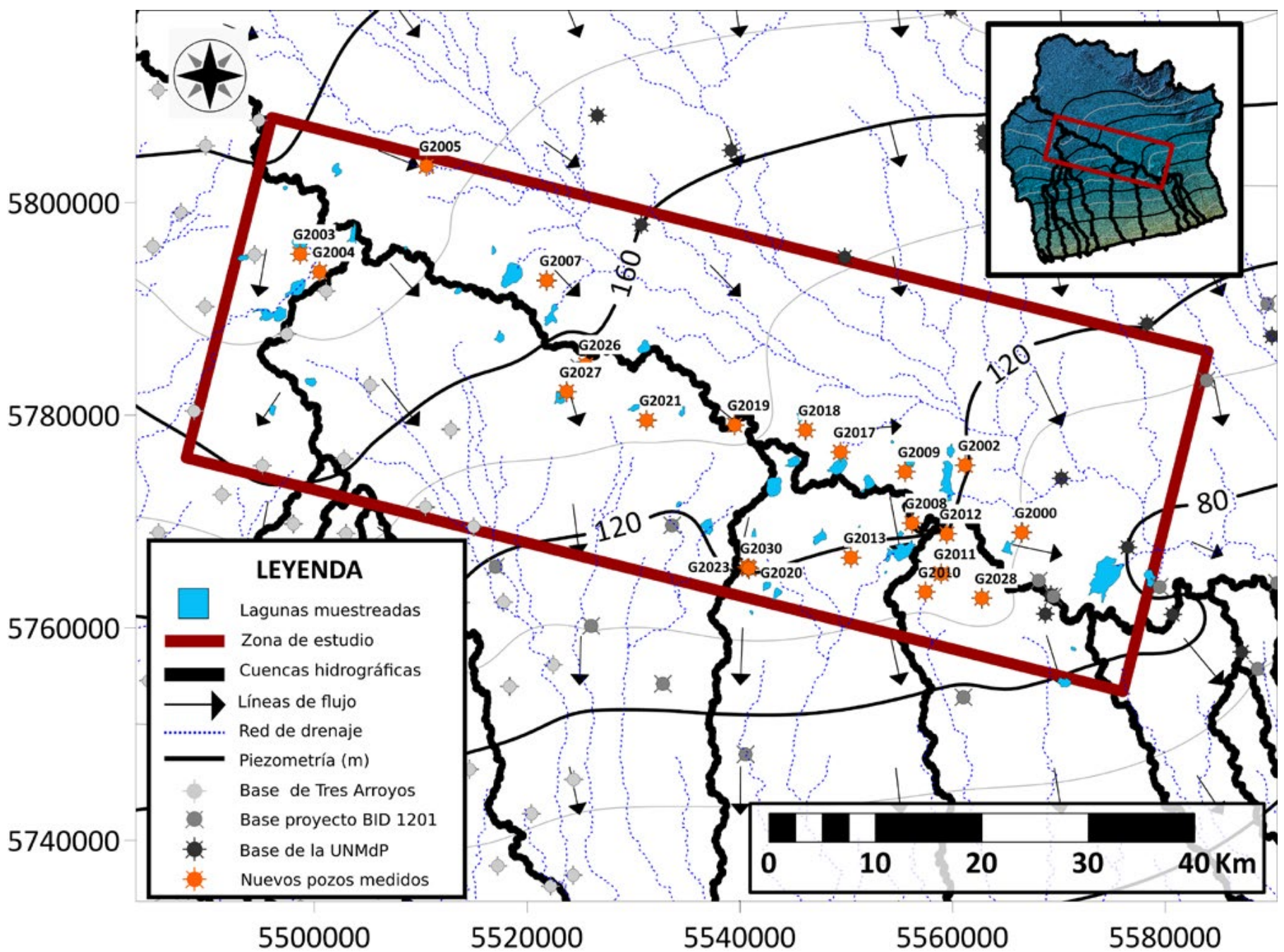

Figura 3. Mapa de nivel piezométrico de la zona de estudio realizado con datos obtenidos entre el 2001 y el 2016.

Figure 3. Piezometric level map of the studied area made with data from 2001 to 2016. 
CHEM (Cambalach y Waterloo Hydrogeologic Inc., 2003) mediante una caracterización estadística general y diagramas convencionales de Piper (Hem, 1992). Las determinaciones isotópicas de $\delta^{2} \mathrm{H}$ y $\delta^{18} \mathrm{O}$ se realizaron con un espectroscopio láser para agua líquida modelo TIWA-45EP, desarrollado por los Gatos Research. La referencia utilizada en el análisis isotópico fue el Vienna Standard Mean Ocean Water (V-SMOW) definida por Gonfiantini (1978), con valores de incertidumbre de $\pm 0,2 \%$ para $\delta^{18} \mathrm{O}$ y $\pm 1,0 \%$ para $\delta^{2} \mathrm{H}$.

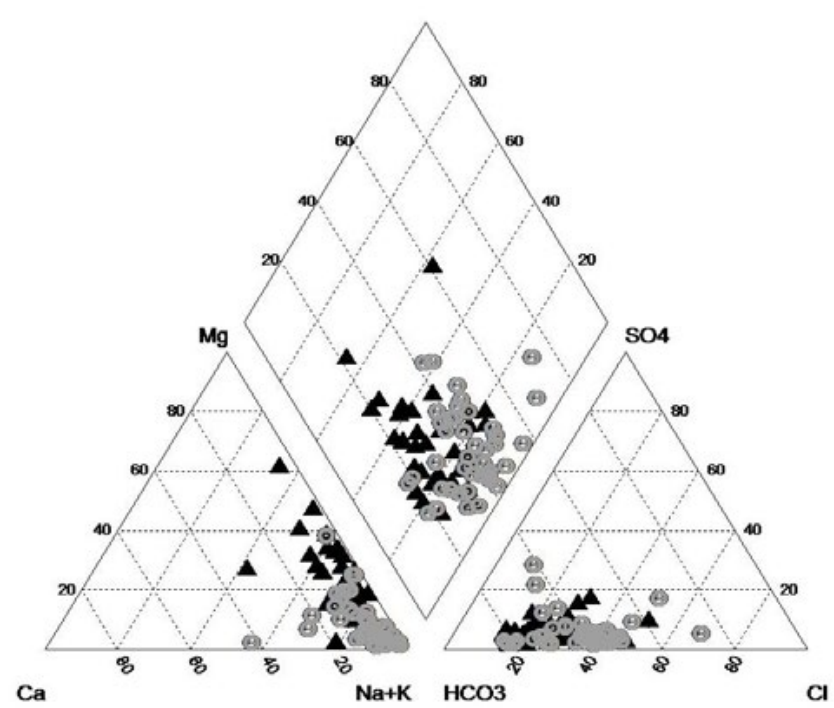

Figura 4. Diagrama de Piper con el contenido iónico de las muestras de agua analizadas.

Figure 4. Piper diagram showing the ionic content of the water samples analyzed.

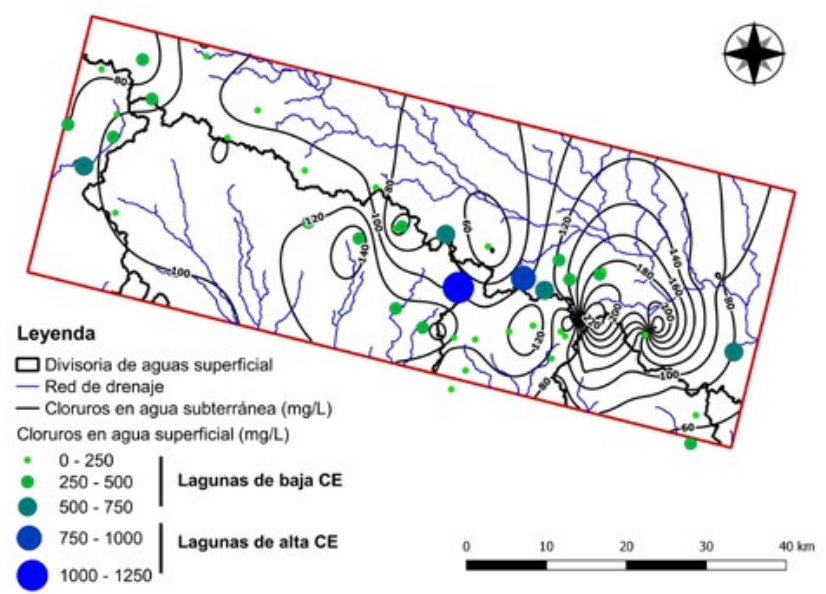

Figura 5. Distribución de cloruros en muestras de agua superficial y subterránea en $\mathrm{mg} / \mathrm{L}$. Las isolíneas se corresponden a las muestras de agua subterránea, mientras que los puntos indican el contenido en cloruros de las muestras de agua de laguna. Se ha añadido la red de drenaje local.

Figure 5. Chloride distribution of groundwater and wetlands ( $\mathrm{mg} / \mathrm{L})$. Contours correspond to groundwater, whilst spots indicate chloride content in the wetland samples. The drainage network is included.

\section{Resultados}

A partir del análisis de los datos de nivel piezométrico recopilados se obtuvo el mapa de la figura 3. En ella se observa una configuración regional del flujo subterráneo con dos direcciones preferenciales. Por un lado, en la CROG el flujo subterráneo sigue una tendencia aproximadamente N-S hasta llegar a la divisoria de aguas superficial en su límite suroccidental, donde se desvía con una dirección NW-SE siguiendo la tendencia del flujo superficial. En lo que se refiere al resto de cuencas, en ellas se mantiene con una tendencia N-S, aproximadamente.

Se representaron los resultados obtenidos en el análisis hidroquímico de las muestras analizadas en un diagrama triangular de Piper (figura 4), observándose que, por lo general, todas las muestras presentan una composición bicarbonatada sódica. En forma general, las muestras de agua subterránea presentan mayor contenido en magnesio que las lagunas y se clasifican como bicarbonatadas sódicas, mientras que las muestras obtenidas de las lagunas aumentan en cloruros.

La variabilidad en la CE de las muestras de agua de los humedales apunta a que existen dos tipos de laguna diferentes, uno de ellos con agua de CE mayores, que podría estar relacionado con el área de descarga de aguas subterráneas con mayor tiempo de tránsito, coincidiendo con zonas de mayor concentración en cloruros (figura 5). El segundo grupo se presenta más bicarbonatado, variando su contenido en magnesio y sodio y con valores de CE más bajos.

Para analizar la composición isotópica de las diferentes fuentes de agua en la zona, se elaboró un diagrama convencional $\delta^{2} \mathrm{H}$ vs. $\delta^{18} \mathrm{O}$, junto con las líneas meteóricas mundial (LMM) y local (LML), reportada por Quiroz et al. (2015) para la CROG y definida por la siguiente ecuación:

$\delta^{2} \mathrm{H} \%$ o $=8,19 \pm(0,14) \cdot \delta^{18} \mathrm{O} \%$ o $13,15 \pm(0,82)$

Ecuación 2: Línea Meteórica Local

En el diagrama de la figura 6 se observa que, por lo general, las muestras de agua subterránea están bien agrupadas alrededor del valor promedio de agua de Iluvia. Este valor fue calculado a partir de las precipitaciones registradas en cuatro estaciones meteorológicas ubicadas a lo largo de la CROG, a partir de las cuales se estableció un valor promedio de -5.14 para $\delta^{18} \mathrm{O} \%$ y -29 para $\delta^{2} \mathrm{H}$. Esta agrupación indica que el acuífero es un sistema bien mezclado, recargado directamente por agua de lluvia. Se encontraron algunas excepciones a este comportamiento, tales como las registradas en los pozos G2040 y G2024, donde el agua se presenta menos empobrecida. Esto sugiere una mezcla entre el agua de lluvia y una fuente menos empobrecida, que según el modelo hidrogeológico conceptual podría deberse a infiltración de agua procedente de las lagunas. Por otro lado, los valores isotópicos de las muestras de laguna se ajustan a una recta de evaporación, indicando que tienen su ori- 


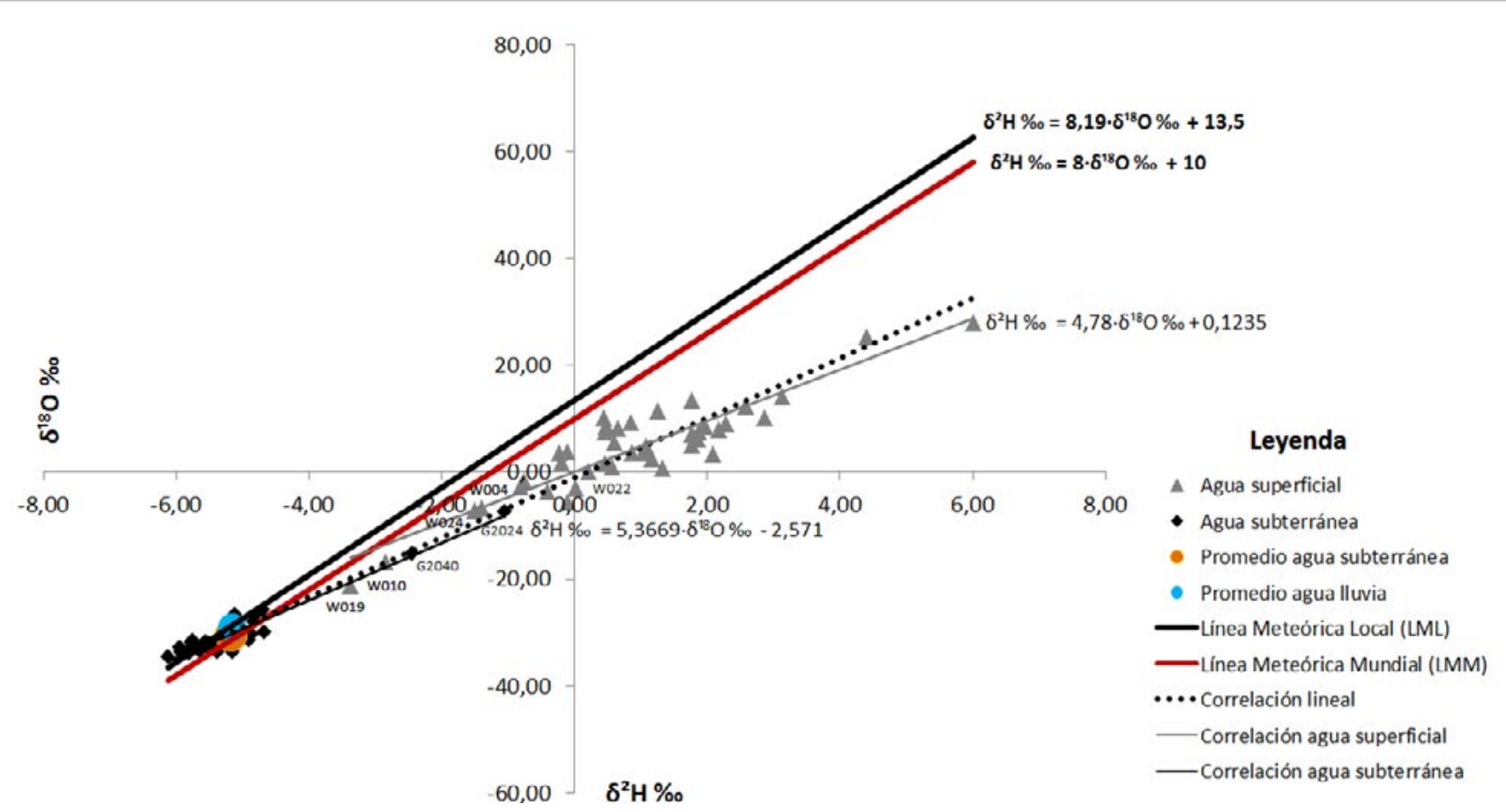

Figura 6. Diagrama $\delta 2 \mathrm{H}$ vs. $\delta 180$ de las muestras de agua superficial y subterránea. Se han representado la LMM, la LML y el promedio de agua de lluvia para la zona. Se han indicado en el gráfico algunas de las lagunas más empobrecidas isotópicamente.

Figure 6. Diagram $\delta 2 \mathrm{H}$ vs. $\delta 180$ of groundwater and wetlands. World Meteoric Line (LMM), Local Meteoric Line (LML) and precipitation average of the studied area are represented.

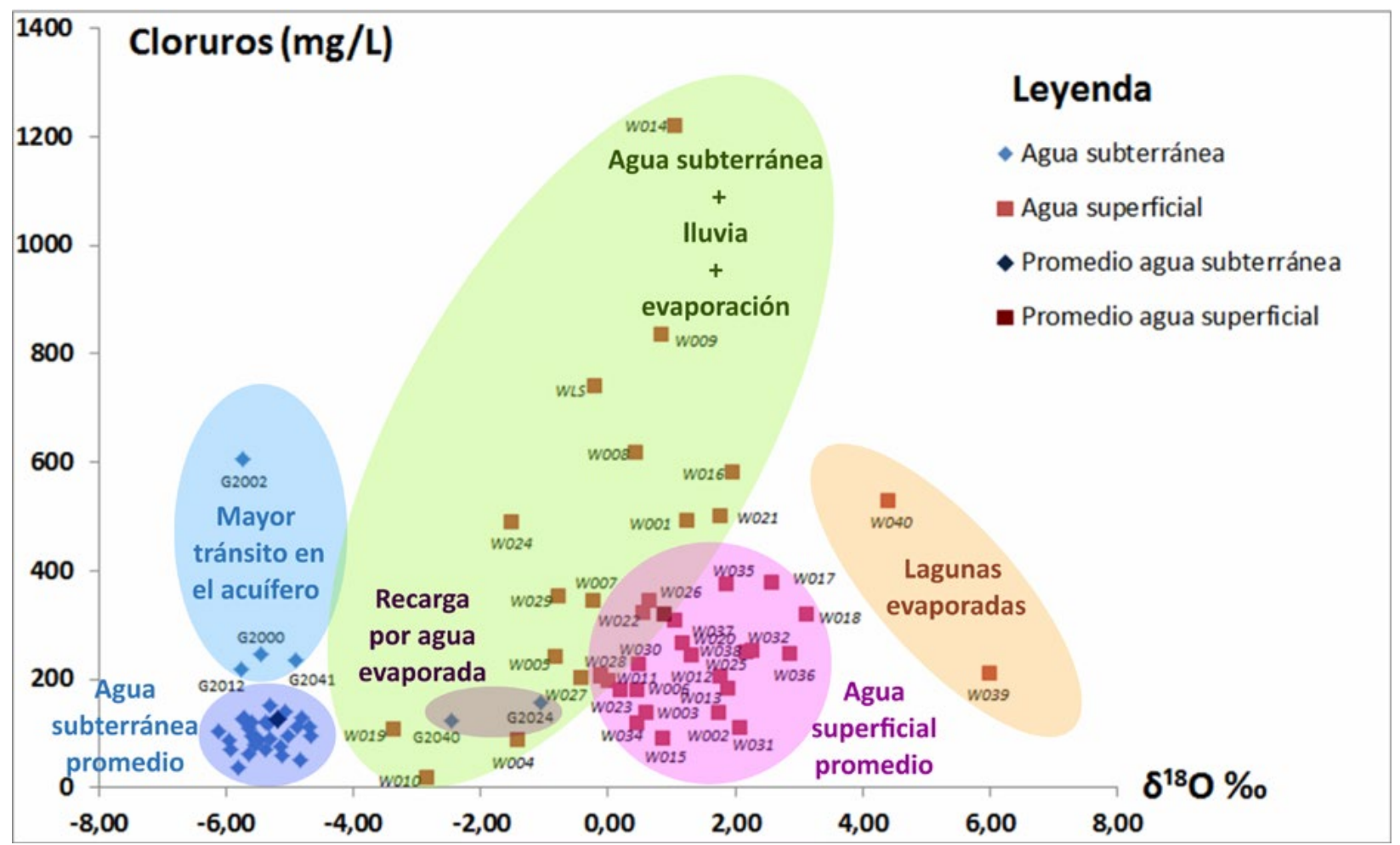

Figura 7. Diagrama $\delta 180$ vs. Cloruros $(\mathrm{mg} / \mathrm{L})$ de las muestras de agua superficial y subterránea. Se han señalado grupos de muestras con comportamiento similar.

Figure 7. $\delta^{18} \mathrm{O}$ versus Chloride $(\mathrm{mg} / \mathrm{L})$ diagram with groundwater and wetland samples. Groups of samples with similar behaviour are differentiated. 
gen en una mezcla de agua subterránea y agua procedente de las precipitaciones con diferentes grados de evaporación. Las muestras de agua de las lagunas más empobrecidas se asocian a procesos de recarga reciente al acuífero, siendo coincidentes con valores empobrecidos de agua de lluvia. Espacialmente, estas lagunas se sitúan al sur del área de análisis y su ubicación coincide con una zona en la que el contenido en $\delta^{18} \mathrm{O}$ del agua subterránea fue relacionado por Quiroz et al. (2015) con el sector noroccidental del sistema serrano de Tandilia. Entre las muestras que describen este comportamiento se encuentra la W010, que fue tomada de una laguna alimentada por una surgencia natural (figura 2).

Some isotopically depleted wetlands are indicated.

Con el propósito de diferenciar grupos de muestras con comportamiento similar, se confeccionó un diagrama $\delta^{18} \mathrm{O}$ vs. contenido en cloruros $(\mathrm{mg} / \mathrm{L}$ ) (figura 7). En el gráfico se observa que el grupo principal de las muestras de agua subterránea se encuentra agrupado y presenta una composición en cloruros que no supera los 200 mg/L. Las muestras G2040 y G2024 conforman otro grupo que mantiene la composición en cloruros, pero son menos empobrecidas en $\delta^{18} \mathrm{O}$. Estas muestras aparecían en el diagrama $\delta^{2} \mathrm{H}$ vs. $\delta^{18} \mathrm{O}$ más alejadas de la composición promedio del agua de lluvia, lo que podría indicar que se recargaron a partir de agua de lluvia evaporada. Sin embargo, su ubicación espacial revela que la recarga procede de agua de laguna infiltrada al acuífero, siendo la laguna W022 la que influye en la composición química del pozo G2024. Un tercer grupo bien diferenciado lo conforman las muestras G2000, G2041, G2012 y G2002, que mantienen una firma isotópica similar al valor promedio pero aumentan en cloruros. Estas muestras se encuentran agrupadas espacialmente y coinciden con la zona este del límite de la CROG (figura 2). Esta correlación puede explicarse de dos maneras: o se trata de aguas con mayor tiempo de tránsito en el acuífero, o bien han estado en contacto con sedimentos ricos en minerales solubles que aporten cloruros, tales como la halita. Dado que no hay evidencias de la existencia de este tipo de depósitos en los sedimentos que conforman el acuífero Pampeano en esta zona, se descarta la segunda posibilidad.

En lo que se refiere a las muestras de las lagunas, el grupo principal presenta una composición en cloruros similar a las muestras de agua subterránea pero están menos empobrecidas en $\delta^{18} \mathrm{O}$, lo que indica que son lagunas evaporadas que se recargan principalmente de agua de lluvia. Un segundo grupo lo conforman las muestras ubicadas en el lado negativo del eje $X$ que, aunque presentan una composición en cloruros variable, su marca isotópica se acerca más a la del agua subterránea, lo que podría estar indicando que se recargan de esta última (figura 7). En el gráfico se han señalado en verde las lagunas con mayor concentración en cloruros y menos empobrecidas en $\delta^{18} \mathrm{O}$, cuyo origen se asocia a una combinación entre aporte de agua subterránea, lluvia y procesos de evaporación. En lo que se refiere a la muestra W040 se ha observado por la red de drenaje que se conecta con la laguna W030, lo que explicaría su desviación en la marca isotópica, siendo agua muy evaporada. Algo similar podría estar sucediendo con la muestra W039.

\section{Conclusiones}

El mapa piezométrico del límite suroccidental de la CROG dejó de manifiesto que el flujo subterráneo se produce en dos direcciones preferenciales, dirigiéndose hacia el sur y hacia el sureste. Este fenómeno podría estar relacionado con la presencia de una barrera hidráulica en profundidad, tratándose de una zona de descarga regional del acuífero Pampeano. Asimismo, se observó que en esta zona, el límite hidrogeológico de la CROG no coincide con la cuenca superficial, ampliándose sus límites subterráneos hacia el sureste y modificándose el modelo hidrogeológico conceptual de la cuenca.

La composición fisicoquímica e isotópica de las lagunas ubicadas en este positivo estructural aportó información relevante al modelo de funcionamiento de la cuenca en este sector, diferenciándose varios grupos de muestras con diferentes marcas isotópicas y comportamiento similar. Ubicándolas espacialmente, se observó una mayor concentración de cloruros tanto en las muestras de agua subterránea como superficial hacia el este del área de estudio.

En cuanto a las características generales de las captaciones de agua, a partir del análisis fisicoquímico e isotópico se obtuvo una composición bicarbonatada sódica predominante para la zona, donde el acuífero Pampeano se recarga esencialmente de agua de lluvia. La interpretación de las desviaciones isotópicas, junto con el contenido en cloruros de las muestras, reveló dos orígenes para los cuerpos de agua superficial. En el grupo principal de lagunas se obtuvo una mezcla de agua subterránea y precipitaciones registradas en el área con diferentes grados de evaporación. Un segundo grupo más empobrecido podría estar indicando procesos de recarga reciente al acuífero, donde el contenido en $\delta^{18} \mathrm{O}$ del agua subterránea se relaciona con el sector noroccidental del sistema serrano de Tandilia. Estas lagunas presentan, al mismo tiempo, valores de CE más altos, lo que sugiere tiempos de tránsito en el acuífero más prolongados.

\section{Referencias}

APHA, 1992. Standard methods for the examination of water and wastewater. 18th ed. American Public Health Association, Washington DC, $1100 \mathrm{pp}$.

Calmbach y Waterloo Hydrogeologic Inc., 2003. Water Quality Data Analysis, Plotting and Modeling. Aquachem User's Manual v 4.0, Co-Developed by Lukas Calmbach and Waterloo Hydrogeologic Inc., United States of America, 276pp. 
Cortizo, L.C. e Isla, F. I., 2000. Land-Cover Change and cliff retreat along the coasts of Necochea and Lobería, Argentina. 9. Simposio Latinoamericano de Percepción Remota Puerto Iguazú, Formato CD 525-533 p., Misiones.

Gat, J. R., 1996. Oxygen and hydrogen isotopes in the hydrologic cycle. Annual Review of Earth and Planetary Sciences, 24(1), 225-262.

Gibson, J. J., Edwards, T. W. D., Birks, S. J., St Amour, N. A., Buhay, W. M., McEachern, P., Wolfe, B. \& Peters, D. L., 2005. Progress in isotope tracer hydrology in Canada. Hydrological Processes: An International Journal, 19(1), 303-327.

Gonfiantini, R., 1978. Standards for stable isotope measurements in natural compounds. Nature 271, 534-536.

Hem, J. D., 1992. Study and interpretation of the Chemical Characteristics of Natural Waters. U.S.G.S. Water-Supply Paper, 2254, fourth printing, 263 pp.

Kendall, C. \& McDonnell, J. J. (Eds.), 2012. Isotope tracers in catchment hydrology. Elsevier.

Kruse, E. Laurencena, P. Deluchi, M. y Varela, I., 1997. Caracterización de la Red de Drenaje para la Evacuación Hidrológica en la Región Interserrana (Provincia de Buenos Aires). En: Actas del I Congreso Nacional de Hidrogeología y II Seminario Hispano - Argentino sobre Temas Actuales de Hidrología Subterránea. Bahía Blanca, 133-145.

Lesta, P. y Sylwan, C., 2005. Cuenca de Claromecó. En: Frontera exploratoria de la Argentina, 6 Congreso de Exploración y Desarrollo de Hidrocarburos (Vol. 10, pp. 217-231).

Llambías, E. y Prozzi, C.R., 1975. Ventania. En: Geología de la Provincia de Buenos Aires, VI Congreso Geológico Argentino, Relatorio 79-101, Buenos Aires.

Martínez, G. A., 2007. Cartografía geomorfológica con imágenes Landsat 7 y Radarsat 1 de la Cuenca del Río Quequén Grande, Provincia de Buenos Aires, Argentina. Teledetección-Herramienta para la gestión sostenible. Ed. Martín, 2007. XII Congreso de la Asociación Española de Teledetección. 19 al 21 de septiembre de 2007. Mar del Plata. 5 pp.

Martínez GA, Quiroz Londoño OM, Martínez DE, Massone HE, Farenga M, Grondona S., 2011. Control neotectónico en la evolución del relieve de la interserrana Bonaerense. XVIII Congreso Geológico Argentino; Neuquén, Argentina. p. 1225-1226.
Martínez, D. Quiroz Londoño, O.M. Dapeña, C. Massone, H.E. Ferrante, A. y Bocanegra, E., 2007. Aportes al modelo hidrogeológico conceptual de la Cuenca del Río Quequén Grande, provincia de Buenos Aires. $V$ Congreso de Hidrogeología y III Seminario hispanoamericano de Temas Actuales de la Hidrogeología Subterránea. Paraná, Entre Ríos, Argentina. 16 - 19 de octubre de 2007. Págs 262 - 271. ISBN 978-987-23936-3-2.

Quiroz Londoño, O. M., Martínez, D., Dapeña, C., y Massone, H., 2008. Hydrogeochemistry and isotope analyses used to determine groundwater recharge and flow in low-gradient catchments of the province of Buenos Aires, Argentina. Hydrogeology Journal, 16(6), 1113-1127.

Quiroz Londoño, O. M., 2009. Tesis doctoral Hidrogeología e Hidrogeoquímica de las Cuencas de los Arroyos Tamangueyú y EI Moro, Provincia de Buenos Aires. Doctorado en ciencias geológicas. Universidad nacional de Rio IV. Córdoba - Argentina.

Quiroz Londoño, O. M., Martínez, D. E., Massone, H. E., Londoño Ciro, L. A., y Dapeña, C., 2015. Spatial distribution of electrical conductivity and stable isotopes in groundwater in large catchments: a geostatistical approach in the Quequén Grande River catchment, Argentina. Isotopes in environmental and health studies, 51(3): 411-425.

Rossello, E. A. (2016). La fracturación del borde oriental de las Sierras Australes de Buenos Aires y su potencial como reservorio de fluidos en la adyacente Cuenca Claromencó. Revista de la Asociación Geológica Argentina, 73(4): 493-512.

Sala, J. M., 1975. Recursos Hídricos (Especial Mención de las Aguas Subterráneas), Relatorio VI Con. Geol. Arg., 169-193.

Solana, M. X., Quiroz Londoño, O. M., \& Martinez, D., 2016. Evolución y origen de lagunas en la Llanura Interserrana mediante el uso de trazadores y sensores remotos. En: IX Congreso Argentino de Hidrogeología y VII Seminario Hispano-Latinoamericano Sobre Temas Actuales de la Hidrología Subterránea, Catamarca. ISBN: 978-987-661-225-8.

Varni, M., Weinzettel, P., \& Usunoff, E., 2006. Hidrogeología del Partido de Tres Arroyos, Provincia de Buenos Aires. Azul (prov. de Buenos Aires). IHLLA. Informe Técnico. Azul, Buenos Aires.
Recibido: julio 2019

Revisado: noviembre 2019

Aceptado: enero 2020

Publicado: marzo 2021 
